\title{
Eating disorders, DSM-5 and clinical reality
}

\author{
Christopher G. Fairburn and Zafra Cooper
}

\begin{abstract}
Summary
The DSM-IV scheme for classifying eating disorders is a poor reflection of clinical reality. In adults it recognises two conditions, anorexia nervosa and bulimia nervosa, yet these states are merely two presentations among many. As a consequence, at least half the cases seen in clinical practice are relegated to the residual diagnosis 'eating disorder not otherwise specified'. The changes proposed for DSM-5 will only partially succeed in correcting this shortcoming. With DSM-6 in mind, it is clear that
\end{abstract}

comprehensive transdiagnostic samples need to be studied with data collected on their current state, course and response to treatment. Only with such data will it be possible to derive an empirically based classificatory scheme that is both rooted in clinical reality and of value to clinicians.

\section{Declaration of interest}

None.
We classify what we know and herein lies the rub as we now know that eating disorders in adults are far more varied and variable than the DSM-IV scheme ${ }^{1}$ suggests. The various iterations of DSM have focused on the two classic clinical presentations that have been highlighted by experts working in specialist treatment centres. $^{2-4}$ The emphasis was initially on anorexia nervosa, with the diagnosis 'bulimia' (later renamed bulimia nervosa) being added in $1980 .{ }^{5}$ Over the intervening years little has changed other than some fine-tuning of the two diagnostic concepts. Meanwhile eating disorder services have moved out of the hospital and into the community, reflecting the fact that the great majority of patients can now be managed on an out-patient basis. With this move has come the realisation that anorexia nervosa and bulimia nervosa are simply two presentations among many. The challenge, and opportunity, for those developing DSM-5 is therefore to think afresh about the classification of the eating disorders and recast the diagnostic scheme in such a way that it accurately represents clinical reality and is of value to clinicians.

\section{The DSM-IV scheme}

The DSM-IV recognises two eating disorders in adults, anorexia nervosa and bulimia nervosa. In addition, there is a 'not otherwise specified' (NOS) diagnosis reserved for eating disorders of clinical severity that do not meet the diagnostic criteria for anorexia nervosa or bulimia nervosa. Thus, in common with the other NOS diagnoses in DSM-IV, eating disorder NOS is a residual category.

Under the rubric of eating disorder NOS, DSM-IV lists a state termed 'binge eating disorder'. This provisional new diagnosis (described in the Appendix 'Criteria sets and axes provided for further study') refers to a clinical picture characterised by recurrent binge eating in the absence of the extreme weightcontrol behaviour seen in anorexia nervosa and bulimia nervosa (e.g. self-induced vomiting, laxative misuse, marked overexercising). The research on binge eating disorder indicates that it is rather different from anorexia nervosa and bulimia nervosa, and indeed from the many other presentations relegated to eating disorder NOS. ${ }^{6,7}$ Those affected tend to be middle aged rather than adolescents or young adults, the marked preponderance of females is absent, and the state commonly co-occurs with obesity. Furthermore, in marked contrast to anorexia nervosa and bulimia nervosa, binge eating disorder tends to be intermittent rather than continuous with many individuals reporting sustained periods when they are free from binge eating.

\section{The problem with the DSM-IV scheme}

The major shortcoming of the DSM-IV scheme for classifying eating disorders is that the supposedly 'residual' diagnosis, eating disorder NOS, is the most common one made in routine clinical practice. Typically about half the cases attract this diagnosis. ${ }^{8-10}$ Apart from those with binge eating disorder, almost all these individuals have the clinical features seen in anorexia nervosa and bulimia nervosa, albeit at slightly different levels and in different combinations. ${ }^{10,11}$ Thus, most show the characteristic overevaluation of shape and weight and its expressions (repeated body checking or body avoidance, extreme feelings of fatness), sustained attempts to follow demanding dietary rules, and weight-control behaviour of the type described earlier. A subset has episodes of loss of control over eating that vary greatly in size (classified as episodes of objective or subjective binge eating). The range of presentations is striking. The problem is how to classify this large and superficially heterogeneous group of cases. $^{11}$

\section{Two potential solutions}

A number of solutions have been proposed. ${ }^{11}$ The least extreme would be to adjust the DSM-IV scheme rather than make any radical change. More specifically, it would be to relax the diagnostic criteria for anorexia nervosa and bulimia nervosa. This solution is based on the premise that the preponderance of eating disorder NOS cases is the result of the DSM-IV diagnostic criteria for anorexia nervosa and bulimia nervosa being too strict, thereby excluding very similar states.

Done mindfully, loosening the diagnostic criteria for anorexia nervosa and bulimia nervosa has much to commend it so long as the core diagnostic concepts are not materially altered. Many clinicians and researchers have suggested that the DSM-IV criteria ought to be adjusted and invariably this would involve relaxing the current diagnostic rules. Two main suggestions have been made with respect to anorexia nervosa. The first is that the amenorrhoea criterion be dropped as it appears to be redundant. ${ }^{12}$ The second is that the weight threshold be raised, ${ }^{13}$ although only a marginal change could be accommodated without undermining the seemingly fundamental requirement that people with anorexia nervosa be underweight. With regard to bulimia nervosa the main proposal has been to lower the frequency threshold for binge eating and 'purging' (self-induced vomiting or laxative misuse). ${ }^{14}$ 
A more radical step would be to recognise binge eating disorder as a third eating disorder thereby extracting these cases from eating disorder NOS. This too seems reasonable given that we now know that binge eating disorder has a distinctive presentation, distribution and course. It also appears to be more treatment responsive than anorexia nervosa and bulimia nervosa. ${ }^{6}$

These changes are indeed those that are being proposed for DSM $-5{ }^{15}$ with the expectation that they will substantially reduce the proportion of eating disorder NOS cases. Will they succeed? Our clinical experience and data suggest that they will be only partially successful. Table 1 shows operational DSM-IV and DSM-5 diagnoses as applied to 167 consecutive adult patients with an eating disorder (body mass index $>15.0$ ) seen in our catchment area-based clinic. Relaxing the diagnostic criteria for anorexia nervosa and bulimia nervosa, and recognising binge eating disorder, does decrease the proportion of eating disorder NOS cases (from 52.7 to $25.1 \%$ ) but still a quarter receive the diagnosis. This is for two reasons. First, rather than being subthreshold forms of anorexia nervosa or bulimia nervosa, many eating disorder NOS cases are 'mixed' with the features of anorexia nervosa and bulimia nervosa occurring in different combinations. Second, if binge eating is carefully distinguished from more everyday overeating, ${ }^{17}$ our experience is that binge eating disorder is not as common as is widely thought.

\section{Three further solutions}

With the eating disorder NOS problem in mind, Walsh \& Sysko ${ }^{18}$ have suggested expanding still further the DSM-IV definitions of anorexia nervosa, bulimia nervosa and binge eating disorder. Their proposal works, but at a cost. In the patient sample mentioned above it results in the proportion of eating disorder NOS cases falling to $7.2 \%$, a figure that seems more appropriate for a residual diagnosis (Table 1; solution 1). However, the cost is that the diagnostic concepts are loosened to such an extent as to become almost unrecognisable. This may be a step too far.

A different strategy would be to accept the DSM-5 proposals as reasonable and to re-classify the remaining eating disorder NOS cases. This requires examining their characteristics. It emerges that almost all have the overevaluation of shape and weight seen in anorexia nervosa and bulimia nervosa accompanied by sustained attempts to follow extreme dietary rules. However, they differ in the presence and frequency of binge eating (objective and subjective), self-induced vomiting, laxative misuse and overexercising, and the extent to which they succeed in undereating, and as a consequence their weight also varies.

There would seem to be two ways of dealing with these remaining cases. The first would be simply to reclassify them as an additional eating disorder, perhaps termed 'mixed eating disorder. ${ }^{11}$ The existence of mixed states is already acknowledged in DSM-5 as exemplified by the proposal that a diagnosis termed 'mixed anxiety depression' be added to the mood disorders section. ${ }^{15}$ The second option would be to subdivide the cases on the basis of their dominant clinical feature. This produces two groups: one in which there is recurrent self-induced vomiting or laxative misuse, often accompanied by subjective binge eating, and the other principally characterised by attempts to follow extreme dietary rules. The former presentation has already attracted the label 'purging disorder'; ${ }^{19}$ the latter might best be termed 'restrained eating disorder'.

By definition, the first solution totally eliminates eating disorder NOS, whereas the second reduces its prevalence to $7.8 \%$ (Table 1; solutions 2 and 3 respectively). Neither solution comes at much cost other than the addition of either one or two new diagnostic concepts, ones that most clinicians would recognise. Therefore, if the primary goal is to eliminate eating disorder NOS, these solutions succeed.

\section{Towards DSM-6}

It is all too easy to get carried away with nosological niceties of this type. Doing so can divert attention from two important matters. The first is the uncomfortable truth that eating disorders are not stable. The behavioural expressions of the overevaluation of shape and weight change with increasing age and duration of disorder. In the early stages most individuals are successful in their attempts to maintain control over their eating and they manage to lose some weight, but as the months and years pass, and they get older, their control tends to break down and episodes of binge eating develop. As a result they regain some or all of the weight that they have lost.

Eating disorder diagnoses are snapshots in the course of an eating disorder. It is not uncommon to encounter individuals who initially met the diagnostic criteria for anorexia nervosa, then those of bulimia nervosa and now have a mixed state. Technically speaking, they have had three distinct psychiatric disorders (anorexia nervosa, then bulimia nervosa and now eating disorder NOS), whereas both common sense and the individual's subjective experience suggest that they have had a single eating disorder that has evolved over time. There are exceptions to this pattern, of course. Most notably, some individuals manage to maintain strict control over their eating and as a result remain underweight. These individuals with persistent anorexia nervosa are worrying and difficult to treat, and so they stay in our mind, but they are a small minority of those with an eating disorder.

The second point that can get overlooked is the purpose of the whole exercise. The DSM is a clinical classificatory scheme and as such it is designed to facilitate clinical work. ${ }^{15}$ Kendell's dictum

\begin{tabular}{|c|c|c|c|c|c|c|c|}
\hline & $\begin{array}{c}\text { Anorexia } \\
\text { nervosa }\end{array}$ & $\begin{array}{l}\text { Bulimia } \\
\text { nervosa }\end{array}$ & $\begin{array}{c}\text { Eating } \\
\text { disorder NOS }\end{array}$ & $\begin{array}{l}\text { Binge eating } \\
\text { disorder }\end{array}$ & $\begin{array}{l}\text { Mixed eating } \\
\text { disorder }\end{array}$ & $\begin{array}{l}\text { Purging } \\
\text { disorder }\end{array}$ & $\begin{array}{l}\text { Restrained } \\
\text { eating disorder }\end{array}$ \\
\hline DSM-IV & 8.4 & 38.9 & 52.7 & - & - & - & - \\
\hline DSM-V & 28.7 & 38.9 & 25.1 & 7.2 & - & - & - \\
\hline Solution $1^{b}$ & 29.9 & 49.7 & 7.2 & 13.2 & - & - & - \\
\hline Solution $2^{b}$ & 28.7 & 38.9 & 0 & 7.2 & 25.1 & - & - \\
\hline Solution $3^{b}$ & 28.7 & 38.9 & 7.8 & 7.2 & - & 10.8 & 6.6 \\
\hline
\end{tabular}


that 'all diagnostic concepts stand or fall by the strength of the prognostic and therapeutic implications they embody ${ }^{20}$ remains as true today as it was in 1975. Therefore, although the surfeit of eating disorder NOS cases can be whittled down in a variety of ways, as illustrated above, what matters is the clinical utility of the various solutions and, in particular, their relative prognostic and therapeutic implications. Unfortunately, these are not known. This is a consequence of the reification of the concepts in DSM. This leads funding bodies and, as a consequence, researchers to study these preordained states rather than focusing on broader, more comprehensive samples of the type needed to test competing diagnostic criteria. An exception was a recent 'transdiagnostic' treatment trial that recruited any patient with an eating disorder so long as their body mass index was over $17.5 .^{21}$ These patients responded well to a single treatment and, interestingly, whether they attracted the diagnosis of bulimia nervosa or that of eating disorder NOS made little difference to their short- or longer-term outcome, a finding that raises questions about the utility of the distinction. So, perhaps more with DSM-6 rather than DSM-5 in mind, it seems that a priority must be to recruit additional comprehensive samples of this type and obtain data not only on their present state but also on their course and response to treatment. Only with such data will it be possible to derive a diagnostic scheme that is rooted in clinical reality and is of value to clinicians.

Christopher G. Fairburn, DM, FMedSci, FRCPsych, Zafra Cooper, DPhil, Dip Psych Oxford University Department of Psychiatry, Warneford Hospital, Oxford, UK

Correspondence: Christopher G. Fairburn, Oxford University

Department of Psychiatry, Warneford Hospital, Oxford OX3 7JX, UK. Email: credo@medsci.ox.ac.uk

First received 18 Jun 2010, final revision 26 Aug 2010, accepted 9 Sep 2010

\section{Funding}

C.G.F. is a Wellcome Principal Research Fellow and Z.C. is supported by a programme grant from the Wellcome Trust (046386).

\section{Acknowledgements}

We are grateful to the Wellcome Trust for their support and we thank Marianne O'Conno for her help applying the various diagnostic algorithms. C.G.F. would also like to acknowledge the lasting influence of one of his mentors, Robert Kendell.

\section{References}

1 American Psychiatric Association. Diagnostic and Statistical Manual of Mental Disorder (4th edn) (DSM-IV). American Psychiatric Association, 1994.

2 Crisp AH. Anorexia Nervosa: Let Me Be. Academic Press, 1980.

3 Garfinkel PE, Garner DM. Anorexia Nervosa: A Multidimensional Perspective. Brunner/Mazel, 1982.

4 Russell GFM. Bulimia nervosa: an ominous variant of anorexia nervosa. Psychol Med 1979; 9: 429-48.

5 American Psychiatric Association. Diagnostic and Statistical Manual of Mental Disorder (3rd edn) (DSM-IIII). APA, 1980.

6 Grilo CM. Eating and Weight Disorders. Psychology Press, 2006.

7 Wonderlich SA, Gordon KH, Mitchell JE, Crosby RD, Engel SG. The validity and clinical utility of binge eating disorder. Int J Eat Disord 2009; 42: 687-705.

8 Ricca V, Mannucci E, Mezzani B, Di Bernardo M, Zucchi T, Paionni A, et al. Psychopathological and clinical features of outpatients with an eating disorder not otherwise specified. Eat Weight Disord 2001; 6: 157-65.

9 Turner H, Bryant-Waugh R. Eating disorder not otherwise specified (EDNOS): profiles of clients presenting at a community eating disorder service. Eur Eat Disord Rev 2004; 12: 18-26.

10 Fairburn CG, Cooper Z, Bohn K, O'Connor ME, Doll HA, Palmer RL. The severity and status of eating disorder NOS: implications for DSM-V. Behav Res Ther 2007; 45: 1705-15.

11 Fairburn CG, Bohn K. Eating disorder NOS (EDNOS): an example of the troublesome "not otherwise specified" (NOS) category in DSM-IV. Behav Res Ther 2005; 43: 691-701.

12 Attia E, Roberto CA. Should amenorrhea be a diagnostic criterion for anorexia nervosa? Int J Eat Disord 2009; 42: 581-9.

13 Watson TL, Andersen AE. A critical examination of the amenorrhea and weight criteria for diagnosing anorexia nervosa. Acta Psychiatr Scand 2003; 108: $175-82$.

14 Wilson GT, Sysko R. Frequency of binge eating episodes in bulimia nervosa and binge eating disorder: diagnostic considerations. Int J Eat Disord 2009; 42: 603-10.

15 American Psychiatric Association. DSM-5 Development. American Psychiatric Association, 2010 (http://www.dsm5.org) (24/09/2010).

16 Fairburn CG, Cooper Z, O'Connor ME. Eating Disorder Examination (16.0D). In Cognitive Behavior Therapy and Eating Disorders (ed CG Fairburn). Guilford Press, 2008.

17 Cooper Z, Fairburn CG. Refining the definition of binge eating disorder and nonpurging bulimia nervosa. Int J Eat Disord 2003; 34: S89-95.

18 Walsh BT, Sysko R. Broad categories for the diagnosis of eating disorders (BCD-ED): an alternative system for classification. Int J Eat Disord 2009; 42 754-64.

19 Keel PK, Striegel-Moore RH. The validity and clinical utility of purging disorder. Int J Eat Disord 2009; 42: 706-19.

20 Kendell RE. The Role of Diagnosis in Psychiatry. Blackwell, 1975.

21 Fairburn CG, Cooper Z, Doll HA, O'Connor ME, Bohn K, Hawker DM, et al. Transdiagnostic cognitive-behavioral therapy for patients with eating disorders: a two-site trial with 60-week follow-up. Am J Psychiatry 2009; 166 311-9. 